\title{
Respuesta de la estructura fitoplanctónica a las perturbaciones antrópicas de un lago templado
}

\author{
Irma Vila y Rodrigo Pardo
}

Departamento de Ciencias Ecológicas. Facultad de Ciencias. Universidad de Chile. Casilla 653 Santiago. limnolog@uchile.cl

\section{RESUMEN}

El Embalse Rapel ( $\left.34^{\circ} \mathrm{S} ; 71^{\circ} \mathrm{W}\right)$ fue generado en 1968, y la comunidad fitoplanctónica señala los efectos de procesos de eutroficación y posterior contaminación por la recepción de agroquímicos y aguas claras de relave de minería del cobre.

La distribución de las diversas asociaciones fitoplanctónicas es homogénea a lo largo del embalse debido a las características hidrodinámicas del sistema con tiempos de retención entre 12 días y tres meses en las áreas diferentes del embalse y vientos diarios que mantienen una mezcla turbulenta de la columna de agua.

Las microalgas se asocian en tres grupos correspondientes a los períodos de diferente calidad química del agua y las asociaciones aquí descritas señalan que taxa como Microcystis aeruginosa, Oscillatoria sp. y Aulacoseira granulata aunque tolerantes a eutrofía son rapidamente afectadas por cobre. La respuesta al aumento de la concentración de cobre señala una disminución en riqueza de especies y un incremento de Dictyiosphaerium pulchellum, Coelastrum microporum y Pediastrum simplex tolerantes al metal.

Palabras claves: Microalgas, fitoplancton, eutrofización, cobre.

\begin{abstract}
The Rapel reservoir $\left(34^{\circ} \mathrm{S} ; 71^{\circ} \mathrm{W}\right)$ was impounded in 1968 and the phytoplankton community shows the effects of eutrophication processes and later contamination due to the affluence of agrochemicals and copper mining clear water tailings.

Phytoplankton diverse associations distribution along the reservoir is homogenous because of the system 's hydrodynamic characteristics with retention times between 12days and three months at the different areas of the reservoir and daily winds that maintain a turbulent mix of the water column.

Micro algae associate in three groups that correspond to periods of different chemical water quality and the associations here described show that taxa such as Microcystis aeruginosa, Oscillatoria sp., and Aulacoseira granulata although tolerant to eutrophy are rapidly affected by copper. The response to the increase of the copper concentration correspons with a decrease in species richness and with a higher abundance of Dictyiosphaerium pulchellum, Coelastrum microporum and Pediastrum simplex as copper tolerant species.
\end{abstract}

Keywords: Micro algae, phytoplankton, eutrophication, copper.

\section{INTRODUCCIÓN}

Clásicamente ha sido demostrado que la composición de las asociaciones fitoplanctónicas depende en gran medida del clima lumínico y físico químico. Durante los últimos años se ha comprobado además, que las interacciones de variables hidráulicas tales como períodos y profundidad de mezcla y estratificación junto con el tiempo de retención de los sistemas acuáticos serían las variables forzantes de mayor impor- tancia en la dinámica de estas asociaciones (Ford, 1990; Reynolds 1997, 1999 y 2000; Armengol et al., 1999; Straskraba, 1999).

Es el caso de los sistemas límnicos chilenos, los cuales se desarrollan en una franja continental que bordea al Océano Pacífico, comprendida entre dos o tres meridianos como promedio $\left(66^{\circ} 30^{\prime} \mathrm{W}-76^{\circ} 30^{\prime} \mathrm{W}\right)$, esta influencia marítima confiere un clima templado y con variaciones térmicas poco marcadas estacionalmente. (Romero, 1985). Con la excepción de los lagos 


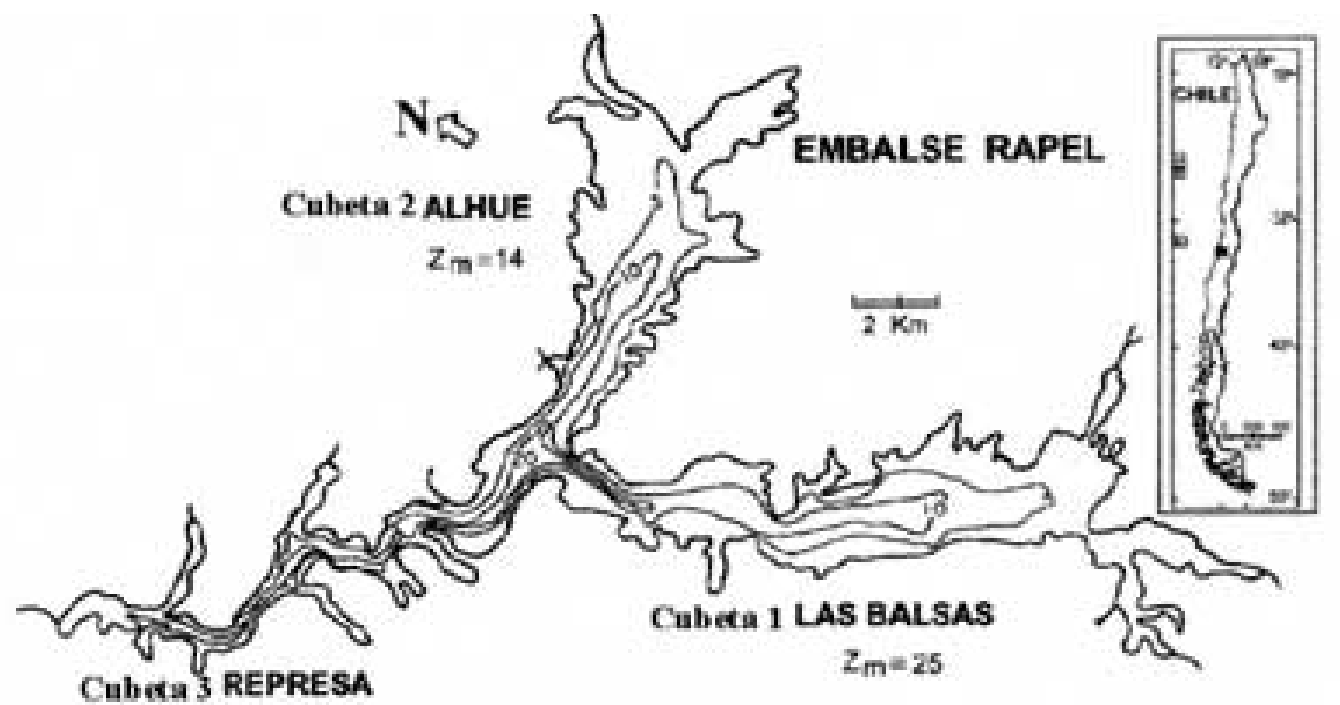

$$
Z_{m}=90
$$

Figura 1. Ubicación geográfica y cubetas del Embalse Rapel. Cubeta 1: Estación fluvial; Cubeta 2 Estación de transición -lacustre y Cubeta 3 Estación represa. Geographic location and basins of the Rapel reservoir. Basin 1: Fluvial station; Basin 2: Transitional station; Basin 3: Dam station.

altoandinos, los lagos ubicados entre los $17^{\circ} \mathrm{S}$ a los $54^{\circ} \mathrm{S}$ se caracterizan por ser monomícticos templados y presentar termoclinas poco estables durante el período de primavera y verano debido a la presencia diaria de vientos SW (Campos, 1984). La mayoría de estos lagos, a semejanza de los sistemas neozelandeses se caracterizan por presentar rangos térmicos reducidos, epilimnion profundo y períodos de estratificación breves (Viner, 1985 y 1987; Geller, 1992). Estas características se acentúan en los embalses por la regulación en la extracción del agua que influiría en el tiempo de residencia hidráulica.

El Embalse Rapel $\left(34^{\circ} \mathrm{S} ; 71^{\circ} \mathrm{W}\right)$ fue generado en 1968, y a ocho años de ser embalsado presentó un proceso de eutroficación acelerado debido a la recepción de agroquímicos y desarrollo rápido del turismo en sus orillas. Las asociaciones de microalgas en este período mostraron la sucesión clásica descrita para embalses templados (DiatomeasClorófitas- Cianófitas) (Hutchinson, 1957; Margalef, 1983; Vila et al., 1987) y con dominancia significativa de Aulacoseira granulata (Ehremberg) Simonsen durante todo el año. Esta especie es muy abundante además, en los lagos del país y de acuerdo con Reynolds (1997) es exitosa en sistemas turbulentos por recuperarse desde los sedimentos. Al incremento de nutrientes por las actividades de turismo y agrícolas de la cuenca se sumó en 1987 el ingreso de aguas claras de relaves de la minería por el cual se incorporó cantidades significativas de sulfatos $\left(1500 \mathrm{mg} \mathrm{L}^{-1}\right)$ asociados principalmente a molibdeno y cobre. Desde la incorporación de sulfatos la dinámica fitoplanctónica se ha visto modificada significativamente al perder la sucesión estacional, disminuir su biomasa y las especies ser reemplazadas por aquellas tolerantes al efecto alguicida del sulfato de cobre (Vila et al., 2000). Sin embargo, durante 1998 se disminuyó la afluencia de sulfatos a $250 \mathrm{mg} \mathrm{L}^{-1}$ y las microalgas señalan nuevos cambios en las asociaciones estacionales.

La distribución de las diversas asociaciones fitoplanctónicas ha sido homogénea a lo largo del embalse y ésto posiblemente sería originado por las características hidrodinámicas del sistema que tiene tiempos de retención entre 12 días y tres meses en las áreas diferentes del embalse, con vientos diarios que mantienen una mezcla turbulenta de la columna de agua (Contreras et al., 1994).

Este trabajo intenta asociar la estructura de las asociaciones fitoplanctónicas como respuesta a 
mecanismos selectivos que generarían las características hidrodinámicas del embalse y el efecto perturbador de sulfatos de molibdeno y cobre en la dinámica de la comunidad fitoplanctónica.

\section{METODOLOGÍA}

El Embalse Rapel ha sido caracterizado con tres zonas o cubetas (Fig. 1). La cubeta 1 corresponde a la zona con características fluviales y profundidades máximas de $25 \mathrm{~m}$. y tiempo de retención $(\tau)$ de 28 días. La cubeta 2 posee características fluvio-lacustres con profundidades máximas de $14 \mathrm{~m}$ y $\tau$ de tres meses. La cubeta 3 , cercana a la presa tiene $\tau$ muy variables y alcanza en la presa $90 \mathrm{~m}$ (Contreras et al., 1994).

Se estudiaron tres períodos anuales 1976-1977, 1990-1991 y 1999-2000 llamados en adelante eutrofización o época inicial, contaminación que corresponde a la máxima incorporación del embalse con sulfatos de cobre con valores de hasta $1500 \mathrm{mg} \mathrm{L}^{-1}$ y recuperación, época durante la cual se restringió el ingreso de sulfato de cobre a $250 \mathrm{mgL}^{-1}$ de sulfato de cobre.

En cada período se obtuvieron muestras de fitoplancton en tres estaciones fijas del Embalse Rapel, las cuales se ubicaron en la zona central de cada una de las cubetas antes descritas. Las profundidades analizadas fueron $0,5 \mathrm{y}$ 10 metros. Simultáneamente, en muestras de agua obtenidas con una botella Van Dorn de tres litros se determinó los valores de clorofila $a$ de acuerdo a Montecino y Cabrera (1982). Se analizó sulfatos con el método turbidimétrico (Golterman et al., 1978).

La composición y abundancia de la comunidad de las microalgas se analizó por microscopía de

Tabla I. Abundancia relativa $>5 \%$ de microalgas durante tres períodos de muestreo. A) Estación represa, B) Estación fluvial y C) Estación fluvio -lacustre. Relative abundance $>5 \%$ of microalgae during three sampled periods. A) Dam station, B) Fluvial station and C)

Intermediate station.

\begin{tabular}{|c|c|c|c|c|c|c|c|c|c|c|c|}
\hline $\mathbf{A}$ & $\begin{array}{c}\text { Dic } \\
1976\end{array}$ & $\begin{array}{c}\text { Mar } \\
1977\end{array}$ & $\begin{array}{c}\text { Jun } \\
1977\end{array}$ & $\begin{array}{l}\text { Ago } \\
1977\end{array}$ & $\begin{array}{c}\text { Jul } \\
1994\end{array}$ & $\begin{array}{c}\text { Ago } \\
1994\end{array}$ & $\begin{array}{c}\text { Dic } \\
1994\end{array}$ & $\begin{array}{c}\text { Mar } \\
1995\end{array}$ & $\begin{array}{c}\text { Mar } \\
2000\end{array}$ & $\begin{array}{c}\text { Jun } \\
2000\end{array}$ & $\begin{array}{l}\text { Ago } \\
2000\end{array}$ \\
\hline \multicolumn{12}{|l|}{ CIANOPHYCEAE } \\
\hline Microcystis aeruginosa (Kütz) & - & 24.46 & - & - & - & - & - & - & - & - & - \\
\hline \multicolumn{12}{|l|}{ Lemmermann } \\
\hline \multicolumn{12}{|l|}{ BACILLARIOPHYCEAE } \\
\hline Aulacoseira granulata (Ehremberg) & 53.78 & 28.52 & 96.49 & 50.63 & 2.49 & 27.96 & 40.32 & 6.68 & 21.44 & 26.84 & 48.75 \\
\hline \multicolumn{12}{|l|}{ Simonsen } \\
\hline Cyclotella menenghiniana Kützing & - & - & - & - & - & - & - & - & 3.48 & 2.88 & 6.25 \\
\hline Cymbella minuta Hilse & - & - & - & - & - & - & - & - & 1.87 & 6.39 & - \\
\hline Navicula sp 3 & - & - & - & 5.48 & - & - & - & - & 0.27 & 0.64 & 1.25 \\
\hline Stephanodiscus astraea (Ehr.) Grun. & - & - & - & - & - & - & - & - & 0.54 & 5.43 & 22.50 \\
\hline Synedra acus Kützing & 2.50 & 4.90 & 0.30 & - & - & - & 0.05 & 21.44 & - & - & - \\
\hline Synedra ulna var. Danica (Kütz.) & 38.58 & 5.03 & 0.19 & - & - & - & - & - & - & - & 1.25 \\
\hline \multicolumn{12}{|l|}{ Grunow } \\
\hline \multicolumn{12}{|l|}{ CHLOROPHYCEAE } \\
\hline Coelastrum microporum Naegeli & 0.16 & 29.14 & 1.37 & - & - & - & 0.37 & 0.30 & 35.93 & 13.42 & 8.75 \\
\hline Dyctiosphaerium pulchellum Naegeli & 0.43 & - & - & - & 95.11 & 63.20 & 13.52 & 1.74 & - & - & - \\
\hline Mougeotia sp. & 2.16 & 1.13 & - & 43.89 & - & - & - & - & - & - & - \\
\hline Pediastrum duplex Meyen & - & - & - & - & - & - & - & - & 16.61 & 7.67 & - \\
\hline Pediastrum simplex Meyen & 1.36 & - & - & - & 1.39 & 7.81 & 45.70 & 47.07 & 1.63 & 1.28 & 6.25 \\
\hline Scenedesmus ecornis (Ralfs) Chodat & - & 6.33 & 0.34 & - & - & - & - & - & 1.07 & 2.56 & 1.25 \\
\hline Staurastrum paradoxum Meyen & 0.05 & - & 0.04 & - & - & - & - & 22.68 & 2.41 & 1.28 & - \\
\hline $\begin{array}{l}\text { Tetraedron minimun (A. Braun) Hansgirg } \\
\text { DINOPHYCEAE }\end{array}$ & - & - & - & - & - & - & - & - & 5.36 & 1.28 & 1.25 \\
\hline Ceratium hirundinella (O.F.M.) Schrank & - & - & - & - & 0.88 & - & - & - & - & 19.17 & - \\
\hline
\end{tabular}


Tabla I. Continuación. Continuation.

\begin{tabular}{|c|c|c|c|c|c|c|c|c|c|c|c|}
\hline B & $\begin{array}{c}\text { Dic } \\
1976\end{array}$ & $\begin{array}{l}\text { Mar } \\
1977\end{array}$ & $\begin{array}{c}\text { Jun } \\
1977\end{array}$ & $\begin{array}{l}\text { Ago } \\
1977\end{array}$ & $\begin{array}{c}\text { Jul } \\
1994\end{array}$ & $\begin{array}{l}\text { Ago } \\
1994\end{array}$ & $\begin{array}{c}\text { Dic } \\
1994\end{array}$ & $\begin{array}{l}\text { Mar } \\
1995\end{array}$ & $\begin{array}{l}\text { Mar } \\
2000\end{array}$ & $\begin{array}{c}\text { Jun } \\
2000\end{array}$ & $\begin{array}{l}\text { Ago } \\
2000\end{array}$ \\
\hline \multicolumn{12}{|l|}{ CIANOPHYCEAE } \\
\hline $\begin{array}{l}\text { Oscillatoria } \text { sp } \\
\text { BACILLARIOPHYCEAE }\end{array}$ & - & 0.38 & 4.02 & 7.59 & - & - & - & - & - & - & - \\
\hline Aulacoseira granulata & 79.46 & 58.78 & 86.33 & 73.18 & - & 4.89 & 20.26 & 10.67 & 59.96 & 34.04 & 37.50 \\
\hline Cyclotella menenghiniana & - & - & - & - & - & - & - & - & 0.41 & 5.32 & 4.17 \\
\hline Ceratoneis araus & - & - & - & - & - & - & - & - & - & - & 8.33 \\
\hline Cymbella minuta & - & - & - & - & - & - & - & - & 0.21 & - & 8.33 \\
\hline Melosira varians & 0.01 & - & - & - & - & 4.33 & - & - & - & - & 10.42 \\
\hline Navicula sp 1 & - & - & - & 13.55 & - & - & - & - & - & - & - \\
\hline Navicula sp 3 & - & - & - & 4.42 & - & - & - & - & - & 4.26 & 31.25 \\
\hline Nitzschia sp. & - & - & - & - & - & - & - & - & - & 13.83 & - \\
\hline Stephanodiscus & - & - & - & - & - & - & - & - & 0.82 & 8.51 & - \\
\hline Synedra acus & 2.90 & 13.98 & - & - & - & 8.20 & 3.80 & 0.16 & - & - & - \\
\hline $\begin{array}{l}\text { Synedra ulna } \\
\text { CHLOROPHYCEAE }\end{array}$ & 12.69 & 5.85 & - & - & - & - & - & - & 0.21 & - & - \\
\hline Closterium acutum & 0.03 & 1.44 & - & - & - & 0.18 & 4.32 & 27.66 & - & - & - \\
\hline Coelastrum microporum & 0.11 & 9.23 & - & - & - & - & - & 0.21 & 7.80 & 8.51 & - \\
\hline Dyctiosphaerium pulchellum & 0.13 & - & - & - & 99.97 & 70.94 & 69.15 & 1.69 & - & - & - \\
\hline Pediastrum simplex & 0.23 & 0.52 & - & - & - & 11.22 & 2.47 & 59.42 & 6.98 & 4.26 & - \\
\hline $\begin{array}{l}\text { Scenedesmus ecornis } \\
\text { DINOPHYCEAE }\end{array}$ & 0.01 & 1.64 & 5.37 & - & - & - & - & - & 3.29 & - & - \\
\hline Ceratium hirundinella & - & - & - & - & - & - & - & - & 5.54 & 12.77 & - \\
\hline $\mathrm{C}$ & $\begin{array}{c}\text { Dic } \\
1976\end{array}$ & $\begin{array}{l}\text { Mar } \\
1977\end{array}$ & $\begin{array}{c}\text { Jun } \\
1977\end{array}$ & $\begin{array}{l}\text { Ago } \\
1977\end{array}$ & $\begin{array}{c}\text { Jul } \\
1994\end{array}$ & $\begin{array}{l}\text { Ago } \\
1994\end{array}$ & $\begin{array}{c}\text { Dic } \\
1994\end{array}$ & $\begin{array}{l}\text { Mar } \\
1995\end{array}$ & $\begin{array}{l}\text { Mar } \\
2000\end{array}$ & $\begin{array}{c}\text { Jun } \\
2000\end{array}$ & $\begin{array}{l}\text { Ago } \\
2000\end{array}$ \\
\hline CIANOPHYCEAE & $* * *$ & & & & & & & & & & \\
\hline Oscillatoria sp 2 & - & 34.96 & 15.98 & 2.14 & - & - & - & - & - & - & - \\
\hline $\begin{array}{l}\text { Microcystis aeruginosa } \\
\text { BACILLARIOPHYCEAE }\end{array}$ & - & 10.61 & 0.08 & - & - & - & - & - & - & - & - \\
\hline Aulacoseira granulata & - & 30.92 & 76.68 & 81.43 & 51.59 & 49.00 & 15.11 & 0.89 & 16.36 & 77.94 & 59.81 \\
\hline Cyclotella menenghiniana & - & - & - & - & - & - & - & - & 4.12 & 3.10 & 13.00 \\
\hline Stephanodiscus & - & - & - & - & - & - & - & - & - & 5.16 & 15.84 \\
\hline $\begin{array}{l}\text { Synedra acus } \\
\text { CHLOROPHYCEAE }\end{array}$ & - & 7.81 & 1.82 & 1.61 & - & - & - & - & - & - & - \\
\hline Coelastrum microporum & - & 0.98 & 0.89 & - & 1.64 & 0.09 & - & 0.06 & 7.08 & 2.84 & 2.36 \\
\hline Dyctiosphaerium pulchellum & - & - & - & - & 11.88 & 0.40 & 2.54 & 0.93 & - & 0.13 & - \\
\hline Mougeotia sp. & - & 7.75 & 0.31 & 11.60 & - & - & - & - & - & - & - \\
\hline Pediastrum simplex & - & - & 0.08 & - & 30.67 & 48.38 & 81.27 & 55.36 & 14.85 & 1.55 & 5.67 \\
\hline Scenedesmus ecornis & - & 1.05 & 0.44 & - & - & - & - & - & 5.03 & - & 0.24 \\
\hline Staurastrum paradoxum & - & 0.42 & 0.03 & - & 0.08 & 0.03 & 0.08 & 42.57 & 1.89 & 0.26 & - \\
\hline $\begin{array}{l}\text { Staurastrum tetracerum } \\
\text { DINOPHYCEAE }\end{array}$ & - & - & 0.05 & - & - & - & - & - & 19.35 & 1.55 & 2.84 \\
\hline Ceratium hirundinella & - & - & - & - & - & - & - & - & 15.73 & 0.13 & - \\
\hline
\end{tabular}

contraste de fase y microscopio invertido OLYMPUS CK2 y se cuantificaron en Unidades Estándar (UE x L-1), representando cada UE $400 \mu^{2}$.
Las comunidades fitoplanctónicas fueron comparadas con análisis de correspondencia de acuerdo con Hair et al., (1992). 


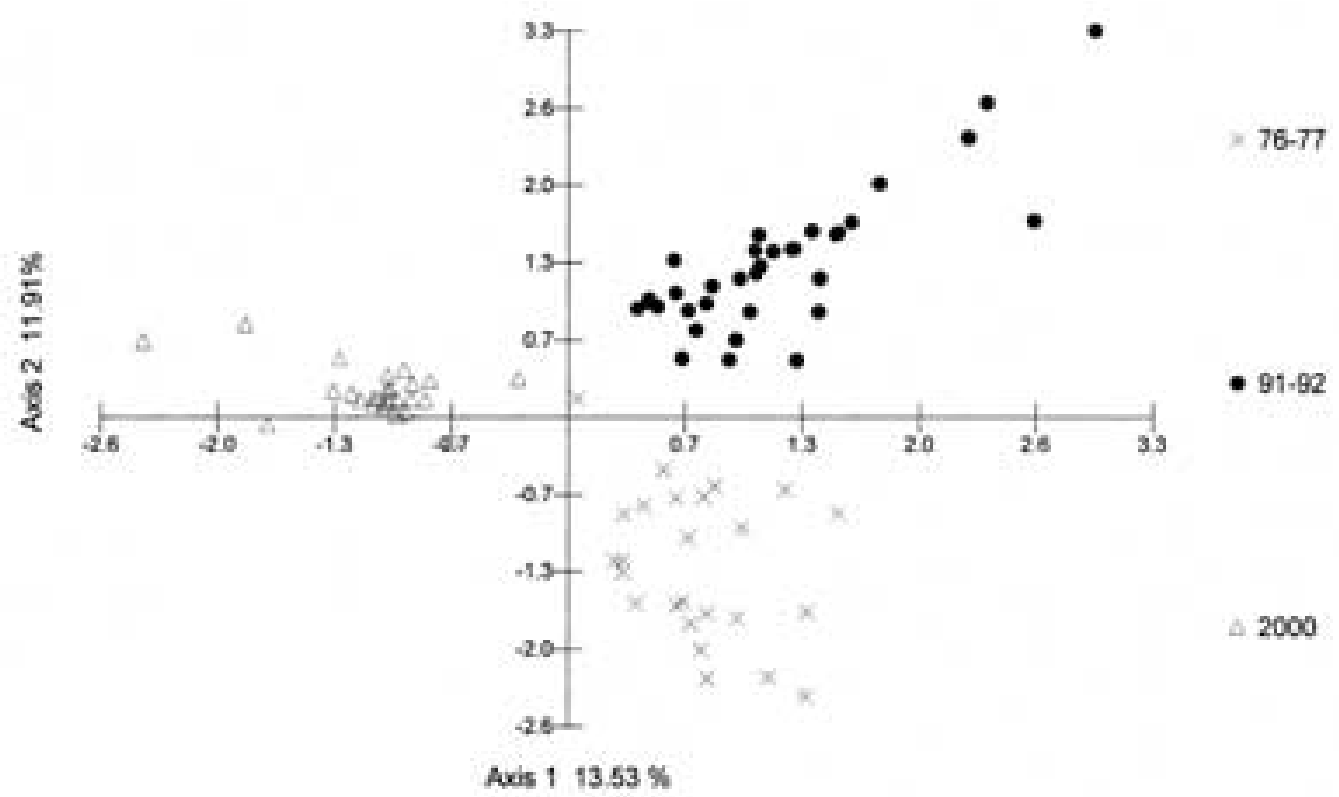

Figura 2. Análisis de correspondencia de las asociaciones de microalgas durante tres períodos en tres cubetas del Embalse Rapel. Correspondence analisys of the microalgal associations during three periods in three basin of the Rapel reservoir.

\section{RESULTADOS}

La Tabla 1a detalla las especies más representativas por período de estudio, en la estación 2 , en el período inicial se contabilizaron 11 especies con más de un $5 \%$ de representación, con dominancia de $A$. granulata. La riqueza disminuyó a siete durante el período de contaminación y dominó Dictyosphaerium pulchellum Naegeli; durante el período de recuperación se encontraron 13 especies, dominando Coelastrum microporum Naegeli y $A$. granulata.

La estación 4 es dominada en la fase inicial por $A$. granulata, encontrándose un total de 12 especies en ese período, luego en el período de contaminación baja el número de especies a siete, con una fuerte dominancia de $D$. pulchellum y al final Pediastrum simplex Meyen. En la fase de recuperación es nuevamente $A$. granulata la especie dominante y se observan 13 especies con más de un $5 \%$ de abundancia (Tabla Ib).

La estación 8 es la que mostró menor numero de especies en todos los períodos (Tabla 1c), observándose 10 especies para la fase inicial, dominando Oscillatoria sp2 y A. granulata, esta última se mantiene como dominante durante todos los muestreos de este período y los primeros dos de la fase de contaminación. En el último muestreo aparece Staurastrum paradoxum Meyen en conjunto con A. granulata, el número de especies encontrado para este período fue de cinco especies. En el período de recuperación se observaron 10 especies, inicialmente la dominancia es compartida entre Staurastrum tetracerum Ralfs y $A$. granulata, en los dos muestreos siguientes es $A$. granulata la especie dominante.

El análisis de correspondencia muestra tanto la similitud en la composición fitoplanctónica a lo largo del embalse en los diferentes períodos estacionales como la diferencia en la organización comunitaria durante los períodos tróficos diferentes del sistema; las microalgas se asocian en tres grupos correspondientes a los períodos de diferente calidad química del agua (Fig. 2). La riqueza promedio de las tres profundidades presentó valores máximos en el período de eutrofización y de recuperación. Durante el período de contaminación, la riqueza se mantie- 


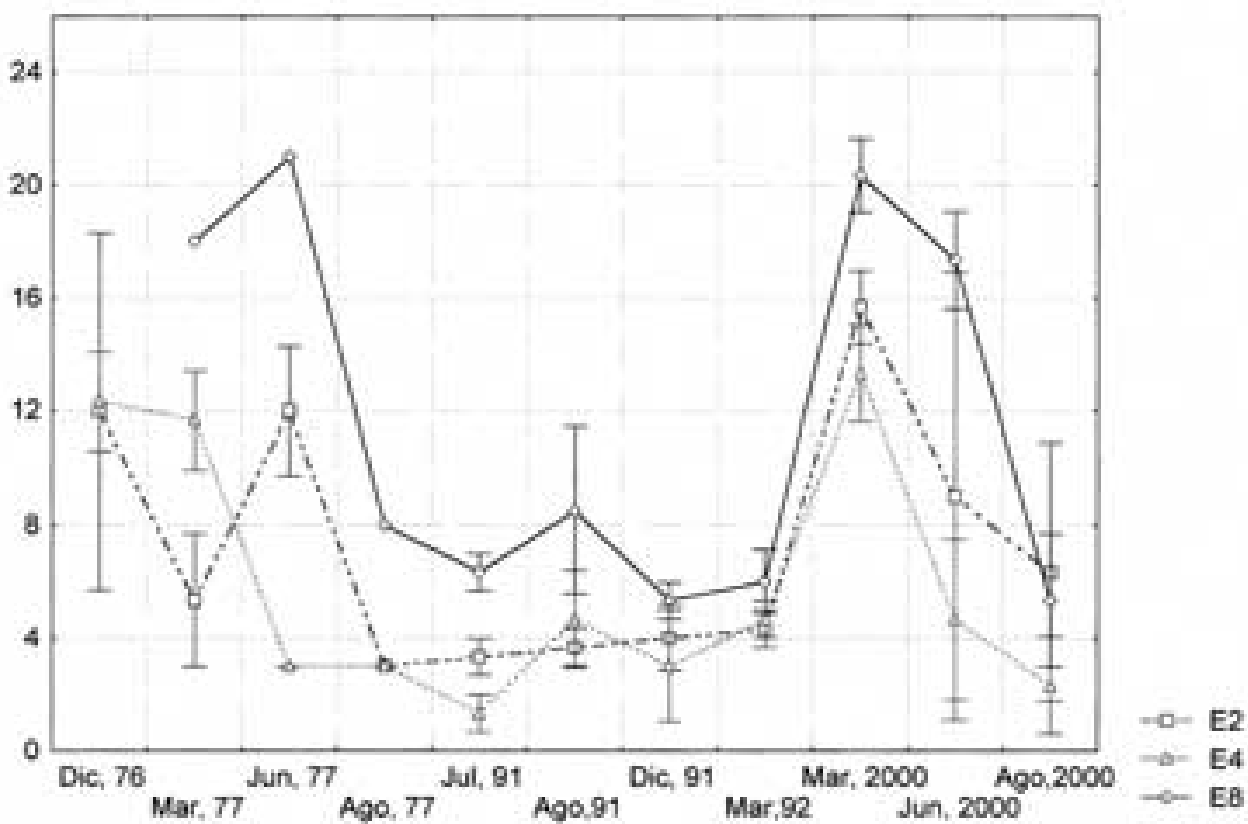

Figura 3. Riqueza de especies durante tres períodos en tres cubetas del Embalse Rapel. Los puntos son el promedio entre tres profundidades y las barras son 1.96 del EE. Species richness during three periods in three basins of the Rapel reservoir. The dots are the average for three depths and the bars indicate 1.96 of SE.

ne en valores bajo ocho especies con un aumento primaveral inferior a los máximos de eutrofización y recuperación (Fig. 3).

Las figuras 4 a - c detallan la evolución de abundancia relativa de $A$. granulata en los tres períodos de estudio. La abundancia superficial de la especie a lo largo del embalse oscila entre el $30 \%$ y el $95 \%$. Esto se repite a las otras profundidades controladas y es la especie dominante. Sus valores de abundancia bajan significativamente en todo el embalse durante la contaminación para alcanzar valores similares de abundancia durante el período de recuperación en la estación fluviolacustre y sobre el $50 \%$ en las otras estaciones. Las variaciones estacionales de abundancia observadas durante el período de eutrofización se enmudecen durante el de contaminación especialmente en lo relacionado con el valor máximo de primavera. La abundancia se incrementa y muestra cambios estacionales durante los últimos años. A. granulata muestra recuperación en la abundancia con respecto al segundo período de estudio, llegando en la Estación fluvio - lacustre a valores similares a los alcanzados en el período de eutrofización. En general las estaciones de trabajo con características más fluviales presentaron las abundancias más homogéneas entre profundidades con la excepción de los meses de Junio y Agosto del año 2000, invierno con lluvias abundantes (Fig. 3).

$\mathrm{La}$ abundancia total en términos de promedios se mantiene similar en los períodos de primavera y verano, sin embargo, con una mayor homogeneidad en el período de contaminación y oscilaciones estacionales amplias en los períodos de eutrofización y de recuperación, ambos períodos mostrando los valores máximos y mínimos encontrados en todo los períodos de estudio. Los valores más bajos durante los tres períodos corresponden a la estación fluvial y los más altos a la estación fluvio-lacustre (Fig. 5).

Los valores de diversidad de ShannonWienner señalan claramente diferencias en los tres períodos de estudio. Durante la eutrofización los valores oscilan entre 0 a 1.7 nits.

Durante el período de contaminación estos valores oscilan entre 0.2 y 1.0 con bastante homogeneidad entre las cubetas. En el período 
A

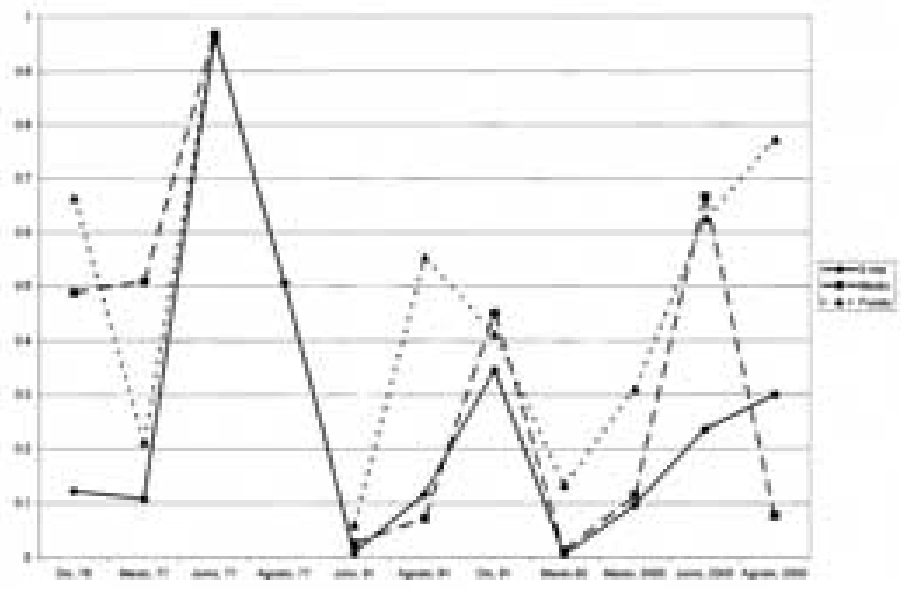

B

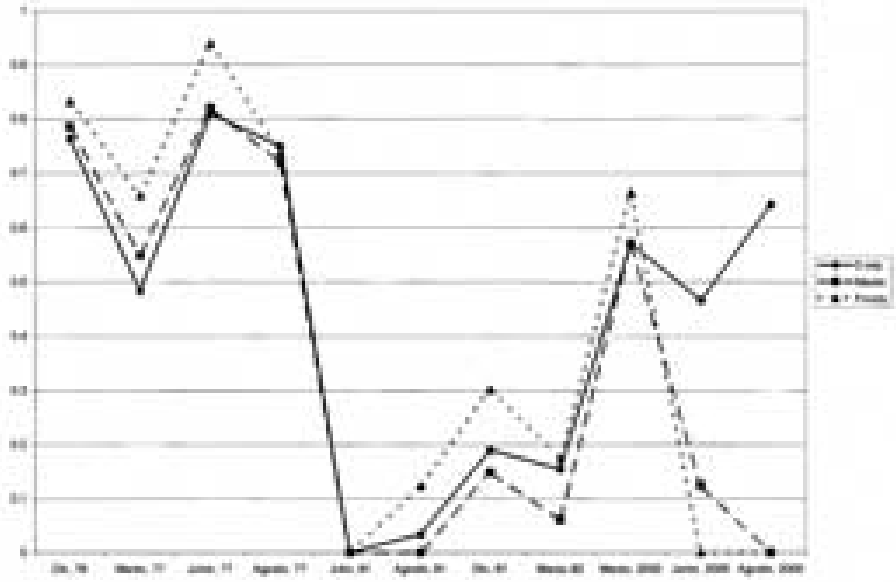

C

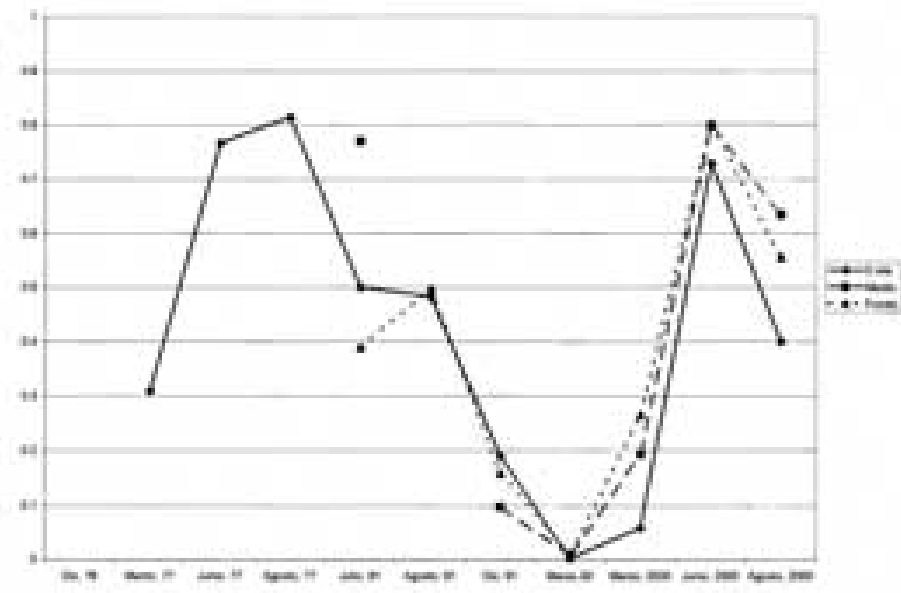

Figura 4. Abundancia relativa de Aulacoseira granulata en los tres períodos de estudio: a superficie, profundidad media y fondo. A) Estación represa, B) Estación fluvial y C) Estación fluvio -lacustre. Relative abundante of Aulacoseira granulata in the three periods of the study: surface, mean depth and bottom. A) Dam station, B) Fluvial station and C) Transitional station. 


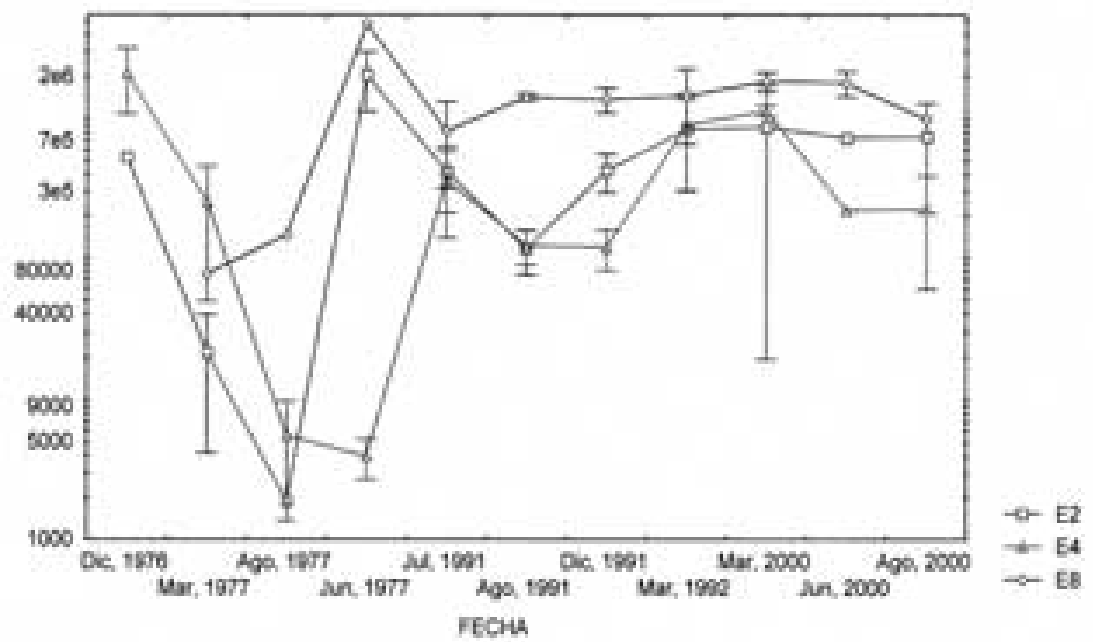

Figura 5. Abundancia total promedio de microalgas entre tres profundidades, a escala logarítmica y barras de 1.96 del EE), en tres períodos. Average total abundance of microalga between three depths, in logarithmic scale and 1.96 SE bars, in three periods.

de recuperación la diversidad oscila entre $1 \mathrm{y}$ 1.8 (Fig. 6). Los valores más bajos de diversidad se estiman para la estación fluvial.

\section{DISCUSIÓN}

Ha sido ampliamente documentada la variabilidad de la composición del fitoplancton en los embalses, especialmente como respuesta a la morfología de los mismos (Kimmel et al., 1990; Reynolds, 1999). En el caso de los sistemas chilenos, a la morfología se suman las variables características de clima oceánico. La mezcla turbulenta casi permanente del agua de los lagos monomícticos templados de Chile, ha favorecido la presencia especies como $A$. granulata, la cual mantiene o incrementa sus poblaciones por resuspensión desde los sedimentos (Reynolds et al., 1985). Bajo condiciones de sucesión normal de los embalses templados, la composición fitoplanctónica ha sido predecible tanto en su composición como su respuesta a los cambios estacionales. En el período inicial de este embalse, la sucesión estacional de las microalgas estaba conformada por especies representativas de la mayoría de los ordenes comunes en estos sistemas. Entre éstas, Chlorophyceae y Cyanophyceae (Margalef, 1983;
Reynolds, 2000), a pesar de la dominancia de $A$. granulata. La disminución marcada de esta especie durante el período de contaminación se explicaría principalmente por el incremento de materia orgánica y sulfatos los cuales estarían produciendo extensas zonas anóxicas cercanas a los sedimentos en las cuales se solubilizarían metales como $\mathrm{Cu}^{+2}$, afectando rápidamente a los inóculos de $A$. granulata. Trabajos realizados con moluscos bentónicos muestran el efecto contaminante de los sulfatos de cobre provenientes de los sedimentos (Valdovinos et al., 1998). En general se menciona que existe información insuficiente acerca de los mecanismos de respuesta a la toxicidad de metales pesados como el cobre y que se requiere de más investigación en el tema y especialmente en aspectos relacionados con las microalgas tanto en sistemas naturales como experimentales. Al respecto, IPCS (1998) resume información que señala que a concentraciones entre $\geq 10 \mathrm{y} \geq 20 \mu \mathrm{g} \mathrm{L}^{-1}$ de cobre, la clorofila $a, \mathrm{y}$ la fijación de nitrógeno y carbono disminuyen significativamente. Filipek et al. (1987) discuten ampliamente y demuestran que las concentraciones de cobre disuelto reflejan la acidez del agua. A pH neutro generalmente la concentración de cobre disuelto está bajo los límites de detección en la columna de agua, pero en los sedimentos, 


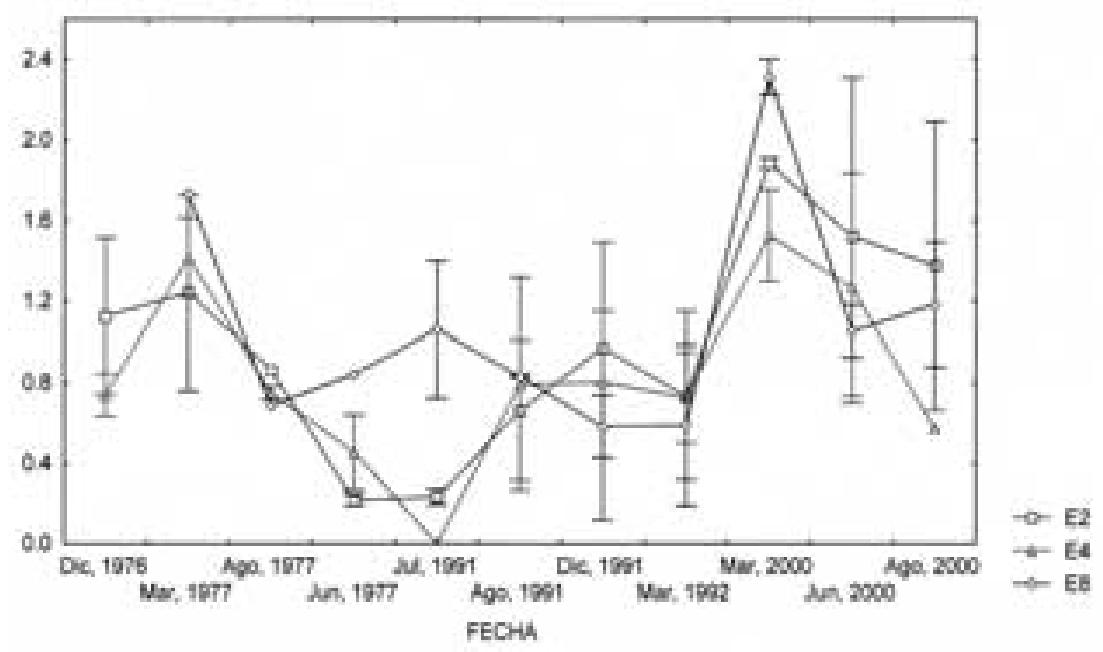

Figura 6. Valores de diversidad de Shannon-Wienner de microalgas en tres cubetas, durante tres períodos. Las barras indican 1.96 de EE. Shannon-Wienner diversity values of microalgae in three basins during three periods. The bars show 1.96 of SE.

que son el depósito y reservorio de metales ocurren cambios diarios y/o estacionales que modifican las interacciones sedimento-agua. Así, aún a pH neutro Winner y Owen (1991) han demostrado que a valores entre $20 \mu \mathrm{g} \mathrm{L}^{-1}$ y $40 \mu \mathrm{g} \mathrm{L}^{-1}$ de cobre se reduce la riqueza de especies de microalgas. Tratamientos con $5 \mu \mathrm{g} \mathrm{L}^{-1}$ de cobre durante 2 a 5 semanas, en Cazenoria, Lake New York, señalaron alteraciones de corto plazo en la sucesión estacional de las microalgas, similarmente a lo ocurrido en el embalse Rapel (Effler et al., 1980; Vila et al., 1997).

Por otra parte, ha sido frecuentemente reportado que la solubilización de $\mathrm{Cu}^{+2}$, afectaría en toda la columna de agua especialmente a las Cyanobacterias descritas como altamente sensibles a este metal (Boyd, 1972; Wurtsbaugh \& Horne, 1982).

La perturbación producida por ralaves de metales como cobre, disminuye significativamente la riqueza de especies en este embalse, sin embargo la presencia de microalgas tolerantes en mayor abundancia apoya la resiliencia del sistema, el cual muestra puntos de compensación en relación con los productores primarios, pero es de importancia evaluar el efecto que los cambios en las asociaciones de las microalgas y la perturbación producirían en la trama trófica del sistema.

\section{BIBLIOGRAFÍA}

ARMENGOL, J., J. C. GARCÍA, M. COMERMA, M. ROMERO, J. DOLZ, M. ROURA, B. H. HAN, A. VIDAL, \& K. SIMEK. 1999. Longitudinal Processes in Canyon Type Reservoirs: The case of Sau (N.E. Spain). In: Theoretical Reservoir Ecology and its applications. J. G Tundisi \& M. Straskraba (eds.): 313-345. International Institute of Ecology. Brazilian Academy of Sciences and Backhuys Publishers.

BOYD, C. 1972. Water quality in warmwater fish ponds. Auburn University, Agricultural Experiment Station, R. Dennis Rouse, Director/Auburn, Alabama.

CAMPOS, H. 1984. Limnological study of Araucanian lakes (Chile). Verh. Internat. Verein. Limnol., 22: 1319-1327.

CONTRERAS, M., H. VILLAGRÁN Y C. SALAZAR. 1994. Características hidrodinámicas del embalse Rapel. Medio Ambiente, 12(1): 41-49.

EFFLER S. W., S. LITTEN, S. D. FIELD, T. TONGNORK, F. HALE, M. MEYER \& M. QUIRK 1980. Whole lake response to low level copper sulphate treatment. Water Res., 14: 1489-1499.

FILIPEK L. H., D. K. NORDSTROM \& W. H. FICKLIN. 1987. Interaction of acid mine drainage with waters and sediments of West Squaw Creek in the west Shasta mining district, California. Environ. Sci. Technol., 21:388-396 
FORD, D. E. 1990. Reservoir transport processes. In: Reservoir Limnology. Ecological perspectives. K. W. Thorton, B. L. Kimmel \& F. E. Payne (eds.):15-41. John Wiley \& Sons Inc. N. Y.

GELLER, W. 1992. The temperature stratification and related characteristics of Chilean lakes in midsummer. Aquatic Sciences, 54: 1-16.

GOLTERMAN, H. L., R. S. CLYMO \& M. A. OHNSTAD. 1978. Methods for physical and chemical analisis of freshwaters. $2^{\text {ond }}$ ed. Blackwell, Oxford.

HAIR, J., R. ANDERSON, R. TATHAM \& W. BLACK. 1992. Multivariate Analysis with Readings. Mac Millan Publishing Co. N. Y.

HUTCHINSON, G. E. 1957. A Treatise on Limnology. Vol. I: Geogrpahy, Physics and Chemistry. N. Y.

IPCS (International Programme on Chemical Safety). 1998. Environmental Health Criteria 2000. Copper. World Health Organization, Geneva.

KIMMEL, B. L., T. L. OWEN \& L. J. PAULSON. 1990. Reservoir Primary Production. In: Reservoir Limnology. Ecological Perspectives. K. W. Thorton, B. L. Kimmel \& F. E. Payne (eds.): 133-194. John Wiley \& Sons Inc. N. Y.

MARGALEF, R. 1983. Limnología. Ed. Omega. Barcelona.

MONTECINO, V. \& S. CABRERA. 1982. Phytoplankton activity and standing crop in an impoundment of Central Chile. J. Plankton Res. 4: 943-950.

REYNOLDS, C., V. MONTECINO, M. E. GRAF \& S. CABRERA. 1985. Short-term dynamics of a Melosira population in the plankton of an impundment in Central Chile. J. Plankton Res. 8: 715-740.

REYNOLDS, C. S. 1989. Physical determinants of phytoplankton succession. In: Plankton Ecology. U. Sommer (ed.): 9-56. Springer. Berlin.

REYNOLDS, C. S. 1997. Vegetation processes in the pelagic: A model for ecosystem theory. Excellence in Ecology, Book 9. Ecology Institute, Nordbunte, Oldendorf/Luhe, Germany.

REYNOLDS, C. S. 1999. Phytoplankton assemblages in Reservoirs. In: Theoretical Reservoir Ecology and its applications. J. G Tundisi \& M. Straskraba (eds.): 439-456. International Institute of Ecology. Brazilian Academy of Sciences and Backhuys Publishers.

REYNOLDS, C. S. 2000. Phytoplankton designer or how to predict compositional responses to trophic state change. Hydrobiologia, 424: 123-132.

ROMERO, H. 1985. Geografía de Chile. Tomo XI: Geografía de los climas. Instituto Geográfico Militar. Santiago, Chile. 243 pp.

STRASKRABA, M. 1999. Retention Time as a Key Variable in Reservoir Limnology. In: Theoretical Reservoir Ecology and its applications. J. G Tundisi \& M. Straskraba (eds.): 385-410. International Institute of Ecology. Brazilian Academy of Sciences and Backhuys Publishers.

VALDOVINOS, C., R. FIGUEROA, H. CID, O. PARRA, E. ARAYA, S. PRIVITERA Y V. OLMOS. 1998. Transplante de organismos bentónicos entre sistemas lénticos: ¿refleja la biodisponibilidad de metales traza en el ambiente? Bol. Soc. Chile. Quim., 43: 467-475.

VILA, I., I. BARENDS \& V. MONTECINO. 1987. Composición, abundancia y distribución espacial y temporal del fitoplancton del embalse Rapel (1976-1977). Revista Chilena de Historia Natural, 60: 37-55.

VILA, I., M. CONTRERAS \& J. PIZARRO. 1997. Eutrophication and phytoplankton selective responses in a temperate reservoir. Verh. Internat. Verein. Limnol., 26: 798-802.

VILA, I., M. CONTRERAS, V. MONTECINO, J. PIZARRO \& D. ADAMS. 2000. Rapel: A 30 years temperate reservoir. Eutrophication or contamination? Arch. Hydrobiol. Spec. Issues Advanc. Limnol., 55: 31-44.

VINER, A. B. 1985. Thermal stability and phytoplankton distribution. In: Perspectives in Southern Hemisphere limnology. R. B. Davies \& R.D. Wamsley (eds.). Dev. Hydrobiol., 28, Dordrecht, Junk.

VINER, A.B. (ed.). 1987. Inland waters of New Zealand. DSIR Bull. 241, Wellington.

WINNER, R.W. \& T. L. OWEN 1991. Seasonal variability in the sensitivity of freshwater phytoplankton communities to a chronic copper stress. Aquat. Toxicol., 19: 73-88.

WURTSBAUGH, W.A., \& A. J. Horne. 1982. Effects of copper on nitrogen fixation and growth of bluegreen algae in natural plankton associations. Can. J. Fish. Aquat. Sci., 39: 1636-1641. 